\title{
3 INTERVENÇÃO FAMILIAR NA ESQUIZOFRENIA: REDUÇÃO DA SOBRECARGA E EMOÇÃO EXPRESSA ${ }^{1}$
}

| Lara Manuela Guedes de Pinho²; Anabela Maria Sousa Pereira ${ }^{3}$ |

\section{RESUMO}

CONTEXTO: A esquizofrenia é uma doença mental grave e crónica que atinge cerca de 1\% da população mundial.

OBJETIVO: implementar um programa de intervenção psicoeducativo em familiares destes doentes e verificar a sua eficácia na redução da emoção expressa e da sobrecarga.

METODOLOGIA: A amostra foi constituída por 20 familiares (10 no grupo experimental e 10 no grupo de controlo). Foi utilizado o Questionário de Problemas Familiares (FPQ) a ambos os grupos, antes e após a intervenção. O grupo experimental foi sujeito a intervenções psicoeducativas multifamiliares enquanto que o grupo de controlo não teve qualquer intervenção.

RESULTADOS: verificou-se a existência de uma sobrecarga objetiva e subjetiva elevada na amostra em estudo, em ambos os grupos, antes da aplicação do programa. Após a aplicação do programa constataram-se diferenças no grupo experimental relativamente a estes fatores, tendo a sobrecarga familiar diminuído. No que respeita à emoção expressa, embora os resultados não tenham sido tão evidentes, houve uma redução do criticismo e aumento das atitudes positivas no grupo experimental. No grupo de controlo não se observaram diferenças no pré e pós teste.

CONCLUSÕES: estes resultados parecem indicar que existiram diferenças possivelmente potenciadas pelo programa, permitindo assim concluir a eficácia da aplicação deste tipo de intervenção, ao nível da diminuição da sobrecarga familiar e da emoção expressa. São referidas algumas implicações deste estudo para a prática clínica e para a intervenção junto dos familiares visando a promoção da saúde e o bem-estar destes indivíduos.

\section{PALAVRAS-CHAVE: Esquizofrenia; Família; Emoção expressa}

\section{RESUMEN}

"Intervención familiar en la esquizofrenia: Reducción de la sobrecarga y emoción expresada"

CONTEXTO: La esquizofrenia es una enfermedad mental grave y crónica que afecta alrededor del $1 \%$ de la población mundial.

OBJETIVO: Implementar un programa de intervención psicoeducativa en la familia y comprobar su eficacia en la reducción de la emoción expresada y sobrecarga.

METODOLOGIA: La muestra está formada por 20 miembros de la familia (grupo experimental (10) y grupo control (10)). Se utilizó el Cuestionario de Problemas de Familia (FPQ) para ambos grupos antes y después de la intervención. El grupo experimental fue sometido a intervenciones psicoeducativas multifamiliares, mientras que el grupo control tuvo ninguna intervención.

RESULTADOS: Se encontró que hay una carga subjetiva y objetiva alta en nuestra muestra, en ambos grupos, antes de la ejecución del programa. Después parece que las diferencias en el grupo experimental de estos factores, y la carga de la familia disminuyeron. En cuanto a la emoción expresada, antes de la ejecución del programa, aunque los resultados no fueron tan evidentes, hubo una reducción de la crítica y el aumento de actitudes positivas en el grupo experimental. En el grupo de control no hubo diferencias en el pre y posttest.

CONCLUSIONES: Estos resultados parecen indicar que existen diferencias posiblemente mejoradas por el programa, lo que confirma la eficacia de la aplicación de este tipo de intervención, a través de la reducción de la carga familiar y expresaron la emoción. Seguirá discutiendo implicaciones de este estudio para la práctica clínica y para la intervención de los familiares destinadas a promover la salud y el bienestar.

\section{DESCRIPTORES: Esquizofrenia; Familia; Emoción expresada}

\section{ABSTRACT \\ "Family intervention in schizophrenia: To reduce burden and expressed emotion"}

CONTEXT: Schizophrenia is a severe chronic mental illness that affects about $1 \%$ of the world population.

OBJECTIVE: The implementation of a psycho-educational protocol with families of schizophrenic patients aiming to evaluate its effectiveness in reducing expressed emotion and family burden.

METHODOLOGY: The study sample consisted of 20 family members (10 in the experimental group and 10 in the control group). Our study used the Family Problems Questionnaire - FPQ, applied before and after the intervention. The experimental group was subjected to a psycho-educational intervention, while the control group wasn't subject to any intervention besides the treatment as usual.

RESULTS: The results indicated the existence of an objective and subjective overload, in both groups, before and after the program, which indicated a high family burden. Regarding the expressed emotion, before the program, although the results were not conclusive, there was a decrease in criticism and increase in the positive attitudes in the experimental group. The control group showed no significant changes.

CONCLUSION: This results suggest that there are differences possibly attributable to the implemented program. Therefore, we can conclude that the implementation of a family psycho-educational program is effective as it potentially decreases family burden and expressed emotion. This study also has implications for clinical practice and to the family intervention aiming at health promotion and the wellness in this area.

\section{KEYWORDS: Schizophrenia; Family; Expressed emotion}

Submetido em 13-09-2014

Aceite em 20-06-2015

1 Artigo extraído da Dissertação de Mestrado em Psicologia Clínica e da Saúde com o título "Esquizofrenia: Eficácia de um programa de psicoeducação familiar", apresentada na Universidade de Aveiro, 2013.

2 Mestre em Psicologia Clínica e da Saúde; Enfermeira Especialista em Enfermagem de Saúde Mental e Psiquiatria no Centro Hospitalar Baixo Vouga, EPE, Departamento de Psiquiatria e Saúde Mental, Avenida Artur Ravara, 3814-501 Aveiro, Portugal, larapinho7@gmail.com

3 PhD em Psicologia; Professora Associada com Agregação na Universidade de Aveiro, Campus Universitário de Santiago, Departamento de Educação, anabelapereira@ua.pt

Citação: Pinho, L. M. G., \& Pereira, A. M. S. (2015). Intervenção familiar na esquizofrenia: Redução da sobrecarga e emoção expressa. Revista Portuguesa de Enfermagem de Saúde Mental (14), 15-23. 


\section{INTRODUÇÃO}

As perturbações mentais são responsáveis por mais de $12 \%$ da carga global de doença a nível mundial, sendo de 23\% nos países desenvolvidos. Além disso, correspondem a 5 das 10 principais causas de incapacidade a longo prazo e de dependência psicossocial, ocupando a esquizofrenia 2,8\% (Xavier, Baptista, Mendes, Magalhães e Caldas-de-Almeida, 2013).

A esquizofrenia é uma perturbação mental grave que apresenta curso e prognóstico variáveis, normalmente sem retorno ao funcionamento pré-mórbido. Os sintomas característicos abrangem uma série de disfunções cognitivas e emocionais e comportamentais que acometem a perceção, o raciocínio lógico, a linguagem e a comunicação, o afeto, a fluência e produção do pensamento e do discurso, a capacidade hedónica, a vontade, o impulso e a atenção. O diagnóstico envolve um conjunto de sinais e sintomas associados a uma disfunção social e ocupacional (American Psychiatric Association, 2014).

Com o início do processo de desinstitucionalização psiquiátrica a responsabilidade dos cuidados no quotidiano dos doentes mentais graves passou a ser da família, na maior parte dos casos sem qualquer preparação. Estima-se que, na comunidade portuguesa, mais de $70 \%$ dos doentes mentais graves não estudam nem trabalham e que 9 em cada 10 doentes com esquizofrenia e em idade ativa vivem numa situação de dependência da sua família (Brito, 2006). A família vivencia uma situação de desgaste psíquico devido a vários fatores como: o impacto do diagnóstico, a necessidade de adaptação a uma nova realidade, o estigma social, a dependência e as implicações da cronicidade da doença psiquiátrica que podem produzir sobrecarga, conflitos, sentimentos de incredulidade, perda do controle, stress e medo de recaídas e do comportamento do doente durante as crises. Assim, pode originar perturbações psiquiátricas, como ansiedade e depressão, que acontecem mais frequentemente nas mulheres que cuidam dos seus filhos ou maridos doentes. Tendo em conta a cronicidade da doença mental, o familiar está sujeito ao efeito prolongado do evento stressor envolvido, na experiência quotidiana de cuidar do doente. Neste sentido, vários estudos têm comprovado que a presença de um doente mental na família origina uma avultada sobrecarga para os seus membros (Borba, Schwartz \& Kantorski, 2008).
A sobrecarga é vista na sua dimensão objetiva e/ou subjetiva. A primeira é mais concreta e de maior intensidade estando relacionada com as causas reais que a convivência com a perturbação mental impõe, enquanto que a sobrecarga subjetiva é abstrata e referese ao universo dos sentimentos (Awad \& Voruganti, 2008; Borba et al., 2008; Leal, Sales, Ibáñez, Giner \& Leal, 2008).

O envolvimento ativo da família no processo de reabilitação do utente com esquizofrenia é de extrema importância.

Como nos indica Reis (2004), foi na década de 70 que emergiu um enorme interesse pelo estudo e intervenção terapêutica nas famílias de doentes psicóticos crónicos, no seguimento dos trabalhos iniciados por George Brown, que comprovou haver maior taxa de recaídas nos doentes que viviam em casa de familiares do que naqueles que viviam sozinhos ou com outros doentes. Esta diferença nas recaídas foi conferida à dimensão emocional das famílias, que mais tarde passou a designar-se por emoção expressa. Verificou-se ainda que quando a emoção expressa das famílias era baixa os doentes recaíam menos do que quando era alta. Yacubian e Neto (2001) referem que utentes com esquizofrenia que vivem com familiares com uma emoção expressa alta tem um risco de recaída duas vezes superior aos que vivem com familiares com uma emoção expressa baixa, no espaço de 9 meses após a alta hospitalar.

As intervenções de psicoeducação familiar têm-se revelado muito eficazes na prevenção de recaídas e dos reinternamentos, contribuindo para melhorar a recuperação das pessoas com esquizofrenia, e para preservar a saúde física e mental dos membros da família, bem como a qualidade de vida (Brito, 2006; Falloon, McGill, Boyd \& Pederson, 1987; GonçalvesPereira, Xavier e Fadden, 2007; Lehman et al., 2004; McFarlane, Dixon, Lukens \& Lucksted, 2003).

A psicoeducação pode definir-se como um conjunto de abordagens que tem como objetivos ajudar os doentes e seus familiares a aprender o que necessitam sobre a doença mental e a dominar novas formas de lidar com ela e com os problemas do quotidiano, bem como reduzir o stress familiar, providenciando suporte social e encorajamento, permitindo um enfoque no futuro mais do que um remoer mórbido do passado (Gonçalves-Pereira, Xavier, Neves, Barahona-Correa e Fadden, 2006; Lucksted, McFarlane, Downing \& Dixon, 2012). 
As intervenções psicoeducativas, no consenso patrocinado pela World Schizophrenia Fellowship, em 1998, contemplam dois objetivos fundamentais: conseguir os melhores resultados clínicos e funcionais para a pessoa com doença mental, através de abordagens que integrem a colaboração entre os profissioais de saúde, famílias e doentes e aliviar o sofrimento dos membros da família, apoiando-os nos seus esforços para potenciar a reabilitação da pessoa que cuidam (Gonçalves-Pereira et al., 2006).

As primeiras abordagens foram criadas enquanto intervenções familiares tendo como objetivo primário a diminuição da taxa elevada de emoção expressa na família ao melhorar o conhecimento da doença e desenvolver as suas competências de gestão da mesma. Têm vindo a desenvolver-se intervenções psicoeducativas centradas na gestão da doença envolvendo diversas abordagens: 1) abordagem com uma família apenas; 2) abordagens multifamiliares com e sem a inclusão das pessoas com esquizofrenia; 3 ) abordagem com as pessoas com esquizofrenia, em formato individual ou em grupo; 4) abordagem bimodal, com as pessoas com esquizofrenia e seus familiares em sessões de grupos separados. Conforme demonstrado em diferentes meta-análises realizadas nos últimos anos, as intervenções psicoeducativas realizadas em grupos de familiares são as mais eficazes em termos quantitativos e qualitativos, não tendo nenhum modelo de intervenção psicológica no tratamento da esquizofrenia evidenciado eficácia superior (Pfammatter, Andres e Brenner, 2012). Mueser e Glynn definiram quatro metas principais da psicoeducação familiar, sendo elas: 1) legitimar a doença mental que visa promover a aceitação familiar da doença, reconhecer os limites que a doença impõe ao doente, desenvolver expectativas realistas e reduzir as responsabilidades do doente; 2) reduzir as emoções negativas dos membros da família, diminuindo a culpa, ansiedade, depressão, raiva e isolamento, mostrar que não estão sozinhos, fornecer um espírito de colaboração na luta contra a doença; 3 ) recrutar a cooperação dos membros da família com o plano de tratamento, explicando intervenções farmacológicas e psicológicas e ajudando os familiares a reforçar a participação do doente no tratamento; 4) melhorar as competências familiares para monitorar a doença, reconhecendo sinais precoces de recaída e mudanças em sintomas persistentes, saber como se comportar para impedir recaídas procurando ajuda profissional e monitorar a adesão ao tratamento e os efeitos secundários da medicação (Yacubian e Neto, 2001).

\section{METODOLOGIA}

\section{Desenho do Estudo}

Trata-se de um estudo experimental de abordagem quantitativa que teve como finalidade avaliar a eficácia de um programa psicoeducativo para familiares de utentes com esquizofrenia usado em contexto hospitalar. A população do estudo são os familiares dos utentes com esquizofrenia de uma unidade hospitalar. A amostra foi constituída, de forma aleatória, por 20 familiares de utentes com o diagnóstico de esquizofrenia (10 no grupo experimental e 10 no grupo de controlo). O protocolo de colheita de dados incluiu uma entrevista estruturada e um instrumento de avaliação, aplicado antes e após a intervenção. Foi realizado um programa de intervenção ao grupo experimental.

\section{Instrumento}

O instrumento de avaliação utilizado foi o FPQ que foi desenvolvido pelo Instituto de Saúde Pública de Roma e pelo Departamento de Psiquiatria da Universidade de Nápoles. Foi traduzido e validado para a versão portuguesa por Miguel Xavier e Caldas de Ameida do Departamento de Saúde Mental da Faculdade de Ciências Médicas de Lisboa. Pretende avaliar a eficácia das intervenções familiares psicoeducativas, sendo constituído por cinco dimensões: sobrecarga objetiva, sobrecarga subjetiva, apoio recebido de profissionais e/ou rede social, atitudes positivas para com o doente e criticismo. Estruturalmente, trata-se de um instrumento de auto-preenchimento constituído por 29 itens obrigatórios (Xavier, Pereira, Corrêa e Caldas-de-Almeida, 2002).

\section{Procedimento}

O estudo obteve parecer positivo da Comissão de Ética da instituição hospitalar. Foram selecionados de forma aleatória utentes com diagnóstico de esquizofrenia, sem outro diagnóstico psiquiátrico associado e constituída a amostra, correspondente aos seus familiares (prestadores de cuidados). Os critérios de exclusão foram a capacidade cognitiva reduzida dos familiares, familiares de utentes que além da doença eram também consumidores de drogas e familiares com doença mental. A recolha de informações que caracterizam a amostra foi realizada através de uma avaliação inicial individual e foram selecionados um grupo de controlo e um grupo experimental, de forma aleatória. 
Depois de esclarecidos os objetivos do estudo, garantida a confidencialidade dos dados auferidos e obtido o consentimento informado, foi aplicado a cada participante o questionário de problemas familiares (FPQ).

Procedeu-se à implementação do programa de psicoeducação familiar no grupo experimental, que teve a duração de 7 sessões, uma vez por semana, em cada grupo, com a duração de aproximadamente 90 minutos, orientadas por um enfermeiro especialista em saúde mental. Esta intervenção decorreu no ano de 2013.

O programa de intervenção utilizado foi um programa multifamiliar de orientação cognitivo-comportamental que comporta os seguintes temas: objetivos da psicoeducação, papel da família como provedora de cuidados, definição de saúde mental e doença mental, definição de psicose/esquizofrenia, sinais e sintomas, causas, etapas e decurso da doença, prevenção de recaídas, estratégias para lidar e agir nos momentos de crise, relacionamento e comunicação familiar, tipos de tratamento, neurobiologia e medicamentos, articulação famílias/profissionais, serviços e recursos disponíveis e lei da saúde mental. Para além da exposição e discussão dos vários temas do programa, foi permitida uma ventilação das emoções, com partilha de experiências, e esclarecimentos de dúvidas. Assim, foi realizada uma espécie de catarse emocional, onde foi permitido aos familiares transmitirem tudo aquilo que sentiam em relação ao seu familiar doente num espaço onde se sentiam compreendidos e onde puderam verificar que não estavam sozinhos, pela partilha de sentimentos entre os vários familiares presentes.

O grupo de controlo não foi sujeito ao programa psicoeducacional.

Foi aplicado novamente o questionário FPQ após 4 meses do início do programa em ambos os grupos (experimental e de controlo).

Após a recolha das informações, realizada através do instrumento que integra o programa de avaliação, efetuou-se o procedimento estatístico dos dados, onde foram realizadas análises descritivas, análises de comparação de grupos (amostras independentes e amostras emparelhadas) e cálculo do tamanho dos efeitos.
As análises descritivas referem-se às variáveis sociodemográficas que tem como objetivo caracterizar a amostra do estudo. Devido ao número reduzido da amostra, recorreu-se à utilização de testes não paramétricos. Para a comparação dos resultados obtidos no pré e pós-teste em ambos os grupos, foi utilizado o teste de Wilcoxon para amostras emparelhadas e para a comparação dos resultados do grupo experimental com o de controlo foi utilizado o Mann-Whitney Test e o cálculo do tamanho dos efeitos foi realizado através do teste não paramétrico delta de Cliff.

\section{Resultados}

As análises descritivas correspondentes às características sociodemográficas da amostra em estudo podem ser visualizadas na tabela 1 .

Tabela 1 - Características Sociodemográficas da Amostra em Estudo

\begin{tabular}{|c|c|c|}
\hline \multirow[b]{2}{*}{ Variáveis } & \multicolumn{2}{|c|}{ Amostra $(n=20)$} \\
\hline & $\begin{array}{l}\text { Grupo Experimental } \\
\qquad(\mathrm{n}=10)\end{array}$ & $\begin{array}{l}\text { Grupo de Controlo } \\
\qquad(\mathrm{n}=10)\end{array}$ \\
\hline Idade & $\mathrm{M}=49,9 ; \mathrm{DP}=14,36$ & $\mathrm{M}=49,1 ; \mathrm{DP}=15,31$ \\
\hline Min-Máx & $18-68$ & $21-69$ \\
\hline Género & & \\
\hline Feminino & $6(60 \%)$ & $10(100 \%)$ \\
\hline Masculino & $4(40 \%)$ & 0 \\
\hline Estado Civil & & \\
\hline Casado [n (\%)] & $6(60 \%)$ & $6(60 \%)$ \\
\hline Divorciado [n (\%)] & $3(30 \%)$ & $3(30 \%)$ \\
\hline Solteiro & $1(10 \%)$ & 0 \\
\hline Viúvo [n (\%)] & 0 & $1(10 \%)$ \\
\hline Habilitações Literárias & & \\
\hline $1^{\circ}$ ciclo (1-4 ${ }^{\circ}$ ano $)[\mathrm{n}(\%)]$ & $2(20 \%)$ & $2(20 \%)$ \\
\hline $2^{\circ}$ ciclo $\left(5-6^{\circ}\right.$ ano $)[\mathrm{n}(\%)]$ & $1(10 \%)$ & $2(20 \%)$ \\
\hline $3^{\circ}$ ciclo $\left(7-9^{\circ}\right.$ ano $)[\mathrm{n}(\%)]$ & $3(30 \%)$ & $1(10 \%)$ \\
\hline $\begin{array}{l}\text { Ensino Secundário }\left(10-12^{\circ}\right. \\
\text { ano) }[\mathrm{n}(\%)]\end{array}$ & $3(30 \%)$ & $4(40 \%)$ \\
\hline Formação Universitária [n(\%)] & $1(10 \%)$ & $1(10 \%)$ \\
\hline Situação Profissional & & \\
\hline Empregado [n (\%)] & $7(70 \%)$ & $6(60 \%)$ \\
\hline Desempregado [n (\%)] & $2(20 \%)$ & $1(10 \%)$ \\
\hline $\begin{array}{l}\text { Aposentado/Reformado [n } \\
(\%)]\end{array}$ & $1(10 \%)$ & $3(30 \%)$ \\
\hline Parentesco & & \\
\hline Pai/Mãe & $6(60 \%)$ & $6(60 \%)$ \\
\hline Cônjuge & $3(30 \%)$ & $1(10 \%)$ \\
\hline Irmãos & $1(10 \%)$ & $1(10 \%)$ \\
\hline Outros & 0 & $2(20 \%)$ \\
\hline
\end{tabular}

Na tabela 2 são apresentadas as características sociodemográficas dos utentes com esquizofrenia, familiares dos participantes que caracterizam a amostra. 
Tabela 2 - Características Sociodemográficas dos Utentes com Esquizofrenia (Familiares da Amostra em Estudo)

\begin{tabular}{|l|l|l|}
\hline \multirow{2}{*}{ Variáveis } & \multicolumn{2}{|c|}{ Amostra (n=20) } \\
\cline { 2 - 3 } & \multicolumn{1}{|c|}{$\begin{array}{c}\text { Grupo Experimental } \\
(\mathrm{n}=10)\end{array}$} & \multicolumn{1}{c|}{$\begin{array}{c}\text { Grupo de Controlo } \\
(\mathrm{n}=10)\end{array}$} \\
\hline Idade & $\begin{array}{l}\mathrm{M}=33,7 ; \mathrm{DP}=14,99 \\
\text { Min-Máx }\end{array}$ & $\begin{array}{l}\mathrm{M}=36,8 ; \mathrm{DP}=11,77 \\
24-59\end{array}$ \\
Género & $19-66$ & $1(10 \%)$ \\
Feminino & $3(30 \%)$ & $9(90 \%)$ \\
Masculino & $7(70 \%)$ & $1(10 \%)$ \\
Estado Civil & & $1(10 \%)$ \\
Casado [n (\%)] & $3(30 \%)$ & $8(80 \%)$ \\
Divorciado [n (\%)] & 0 & $2(20 \%)$ \\
Solteiro & $7(70 \%)$ & $2(20 \%)$ \\
Habilitações Literárias & & $5(50 \%)$ \\
$1^{\circ}$ ciclo (1-4 ano) [n (\%)] & $2(20 \%)$ & $1(10 \%)$ \\
$2^{\circ}$ ciclo (5-6 ano) [n (\%)] & 0 & \\
$3^{\circ}$ ciclo (7-9 ano) [n (\%)] & $5(50 \%)$ & $8(80 \%)$ \\
Ensino Secundário (10-12 & $3(30 \%)$ & $2(20 \%)$ \\
ano) [n (\%)] & & \\
Situação Profissional & $8(80 \%)$ & \\
Desempregado [n (\%)] & $2(20 \%)$ & \\
Reformado por invalidez [n & & \\
(\%)] & & \\
\hline
\end{tabular}

As análises de comparação de grupos efetuadas centraram-se no estudo dos quatro fatores que constituem o questionário, sobrecarga objetiva, sobrecarga subjetiva, atitudes positivas e criticismo. Em relação ao momento antes do programa verificou-se que apenas a variável criticismo apresentou diferenças significativas, sendo que no grupo experimental $(\mathrm{M}=1,70 ; \mathrm{DP}=0,25)$ e no grupo de controlo $(\mathrm{M}=2,23 ; \mathrm{DP}=0,75)$. Verifica-se um nível mais elevado de criticismo no grupo de controlo. Estes resultados estão descritos na tabela 3.

Tabela 3 - Análise das Diferenças entre os Dois Grupos Antes do Programa Psicoeducacional

\begin{tabular}{|l|l|l|l|l|l|}
\hline & $\begin{array}{l}\text { Grupo experimental } \\
(\mathrm{N}=10)\end{array}$ & $\begin{array}{l}\text { Grupo de } \\
\text { controlo } \\
(\mathrm{N}=10)\end{array}$ & $\mathrm{U}$ & $\mathrm{P}$ & $\Delta$ \\
\hline & $\mathrm{M}(\mathrm{DP})$ & $\mathrm{M}(\mathrm{DP})$ & & & \\
\hline $\begin{array}{l}\text { Fator 1 - Sobrecarga } \\
\text { objetiva }\end{array}$ & $2,20(0,54)$ & $2,66(1,15)$ & 48,5 & 0,909 & \\
\hline $\begin{array}{l}\text { Fator 3 - Sobrecarga } \\
\text { subjetiva }\end{array}$ & $2,63(0,65)$ & 46 & 0,762 & \\
\hline $\begin{array}{l}\text { Fator 4 - Atitudes } \\
\text { positivas }\end{array}$ & $2,52(0,55)$ & $2,01(0,43)$ & 24,5 & 0,051 & \\
\hline Fator 5 - Criticismo & $1,70(0,25)$ & $2,23(0,75)$ & 24,5 & 0,045 & $-0,43$ \\
\hline
\end{tabular}

Relativamente ao momento após o programa, como se pode visualizar na tabela 4 , verificou-se que apenas as variáveis atitudes positivas e criticismo apresentaram diferenças estatisticamente significativas, sendo que na variável atitudes positivas no grupo experimental $(M=2,73 ; D P=0,47)$ e no grupo de controlo $(M=2,08$; $\mathrm{DP}=0,52)$ e na variável criticismo no grupo experimental $(M=1,27 ; \mathrm{DP}=0,34)$ e no grupo de controlo $(\mathrm{M}=2,08 ; \mathrm{DP}=0,54)$. Verifica-se um nível mais baixo de atitudes positivas e mais elevado de criticismo no grupo de controlo.
Tabela 4 - Análise das Diferenças entre os Dois Grupos Após o Programa Psicoeducacional

\begin{tabular}{|l|l|l|l|l|l|}
\hline & $\begin{array}{l}\text { Grupo experimental } \\
(\mathrm{N}=10)\end{array}$ & $\begin{array}{l}\text { Grupo de } \\
\text { controlo } \\
(\mathrm{N}=10)\end{array}$ & $\mathrm{U}$ & $\mathrm{P}$ & $\Delta$ \\
\hline & $\mathrm{M}(\mathrm{DP})$ & $\mathrm{M}(\mathrm{DP})$ & & & \\
\hline $\begin{array}{l}\text { Fator 1 - Sobrecarga } \\
\text { objetiva }\end{array}$ & $1,67(0,32)$ & $2,29(0,95)$ & 33 & 0,198 & \\
\hline $\begin{array}{l}\text { Fator 3 - Sobrecarga } \\
\text { subjetiva }\end{array}$ & $2,05(0,38)$ & $2,53(1,02)$ & 43 & 0,591 & \\
\hline $\begin{array}{l}\text { Fator 4 - Atitudes } \\
\text { positivas }\end{array}$ & $2,73(0,47)$ & $2,08(0,52)$ & 16 & 0,010 & 0,60 \\
\hline Fator 5 - Criticismo & $1,27(0,34)$ & $2,08(0,54)$ & 7,5 & 0,001 & $-0,51$ \\
\hline
\end{tabular}

Em relação ao grupo experimental, verificaram-se diferenças estatisticamente significativas com o programa psicoeducacional nas variáveis, sobrecarga objetiva $(\mathrm{p}=0,037)$, sobrecarga subjetiva $(\mathrm{p}=0,007)$ e criticismo $(\mathrm{p}=0,016)$. Especificamente, estas variáveis apresentaram valores superiores antes do programa psicoeducacional do que após o programa, a sobrecarga objetiva passou de $(\mathrm{M}=2,20 ; \mathrm{DP}=0,54)$ para $(\mathrm{M}=1,67 ; \mathrm{DP}=0,32)$; a variável sobrecarga subjetiva diminuiu de $(\mathrm{M}=2,63$; $\mathrm{DP}=0,65)$ para $(\mathrm{M}=2,05 ; \mathrm{DP}=0,38)$ e a variável criticismo alterou de $(M=1,70 ; D P=0,25)$ para $(M=1,27$; $\mathrm{DP}=0,34)$. Estes resultados estão descritos na tabela 5 .

Tabela 5 - Análise das Diferenças Antes e Após a Intervenção no Grupo Experimental

\begin{tabular}{|l|l|l|l|l|l|}
\hline & $\begin{array}{l}\text { Pré-Teste } \\
(\mathrm{N}=10)\end{array}$ & $\begin{array}{l}\text { Pós-Teste } \\
(\mathrm{N}=10)\end{array}$ & $\mathrm{Z}$ & $\mathrm{p}$ & $\Delta$ \\
\hline & $\mathrm{M}(\mathrm{DP})$ & $\mathrm{M}(\mathrm{DP})$ & & & \\
\hline $\begin{array}{l}\text { Fator 1 - Sobrecarga } \\
\text { objetiva }\end{array}$ & $2,20(0,54)$ & $1,67(0,32)$ & $-2,091$ & 0,037 & 0,62 \\
\hline $\begin{array}{l}\text { Fator 3 - Sobrecarga } \\
\text { subjetiva }\end{array}$ & $2,63(0,65)$ & $2,05(0,38)$ & $-2,677$ & 0,007 & 0,31 \\
\hline $\begin{array}{l}\text { Fator 4 - Atitudes } \\
\text { positivas }\end{array}$ & $2,52(0,55)$ & $2,73(0,47)$ & $-1,620$ & 0,105 & \\
\hline Fator 5 - Criticismo & $1,70(0,25)$ & $1,27(0,34)$ & $-2,410$ & 0,016 & 0,20 \\
\hline
\end{tabular}

O grupo de controlo, não sujeito ao programa psicoeducacional, não apresentou diferenças estatisticamente significativas no pré e pós teste, como se visualiza na tabela 6.

Tabela 6 - Análise das Diferenças Antes e Após a Intervenção no Grupo de Controlo

\begin{tabular}{|l|l|l|l|l|}
\hline & $\begin{array}{l}\text { Pré-Teste } \\
\text { (N=10) }\end{array}$ & $\begin{array}{l}\text { Pós-Teste } \\
(\mathrm{N}=10)\end{array}$ & $\mathrm{Z}$ & $\mathrm{p}$ \\
\hline & $\mathrm{M}(\mathrm{DP})$ & $\mathrm{M}(\mathrm{DP})$ & & \\
\hline $\begin{array}{l}\text { Fator 1 - Sobrecarga } \\
\text { objetiva }\end{array}$ & $2,49(0,98)$ & $2,29(0,95)$ & $-1,021$ & 0,307 \\
\hline $\begin{array}{l}\text { Fator 3 - Sobrecarga } \\
\text { subjetiva }\end{array}$ & $2,66(1,15)$ & $2,53(1,02)$ & $-0,841$ & 0,400 \\
\hline Fator 4 - Atitudes positivas & $2,01(0,43)$ & $2,08(0,52)$ & $-0,302$ & 0,763 \\
\hline Fator 5 - Criticismo & $2,23(0,75)$ & $2,08(0,54)$ & $-0,420$ & 0,674 \\
\hline
\end{tabular}


No follow up do estudo, um ano após o final do programa foi avaliado o número de internamentos dos utentes com esquizofrenia (familiares de ambos os grupos), tendo-se constatado que no grupo experimental apenas um utente foi internado com a duração de 21 dias.

Relativamente ao grupo de controlo, seis dos utentes destes familiares tiveram internamentos nesse mesmo período de tempo, sendo que, três foram internados apenas uma vez (11, 14 e 20 dias respetivamente), um foi internado duas vezes ( 35 dias e 26 dias) e dois foram internados três vezes ( $1^{\circ} 13,15$ e 27 dias; $2^{\circ}$ 9, 13 e 16 dias).

\section{DISCUSSÃO}

Com este estudo de investigação pretendeu-se implementar e avaliar a eficácia da aplicação de um programa psicoeducacional em familiares de doentes com esquizofrenia especificamente na sobrecarga familiar e na emoção expressa, bem como no número de internamentos dos utentes.

Em relação à comparação de ambos os grupos antes da aplicação do programa, verificou-se que estes são semelhantes nos fatores sobrecarga objetiva, sobrecarga subjetiva e atitudes positivas, verificando-se diferenças estatisticamente significativas apenas no fator criticismo. Este fator é menor no grupo experimental do que no grupo de controlo.

Analisando estes resultados verificamos que existe uma sobrecarga objetiva e subjetiva elevada na amostra em estudo. Os valores da média nestas variáveis são superiores ao estudo realizado por Xavier et al. (2002), o que nos indica uma maior sobrecarga nos familiares da amostra do presente estudo de investigação. Estes resultados vão de encontro ao esperado, uma vez que vários estudos têm comprovado que a presença de um doente mental na família origina uma avultada sobrecarga para os seus membros (Borba et al., 2008; Brito, 2006).

Após a aplicação do programa, entre os grupos, verifica-se que existem diferenças estatisticamente significativas no fator atitudes positivas e que se mantém diferenças significativas em relação ao fator criticismo. Embora antes da aplicação do programa esta diferença entre os dois grupos, no fator criticismo, já existisse e já fosse menor no grupo experimental, o tamanho do efeito aumentou verificando-se uma diminuição do $\Delta$, de $-0,43$ para $-0,51$, passando de um tamanho pequeno a médio para médio a grande.
Estes resultados parecem indicar que existiram diferenças possivelmente potenciadas pelo programa implementado, uma vez que os sujeitos de ambos os grupos mantiveram todas as intervenções adicionais estáveis e a única alteração foi a participação (ou não participação) no programa. Embora os fatores sobrecarga objetiva e sobrecarga subjetiva não tenham apresentado diferenças significativas estatisticamente comparando ambos os grupos após a intervenção, todos eles apresentaram diferenças nas médias superiores ao momento antes da intervenção, tendo o grupo experimental resultados mais positivos em todos os fatores.

Na comparação do grupo experimental antes e após o programa, apesar do tamanho reduzido da amostra, conseguiram-se verificar melhorias estatisticamente significativas tanto na diminuição da sobrecarga familiar (objetiva e subjetiva) como na diminuição do criticismo. Em termos de interpretação clínica do d de Cohen (alternativa paramétrica do delta de Cliff e a partir da qual, através de uma análise de correspondências, é possível uma interpretação mais completa do tamanho dos efeitos), efeitos a partir de 0,50 significam melhorias clinicamente significativas. Assim, podemos concluir que, em relação à sobrecarga objetiva, ocorreram melhorias clinicamente significativas $(\Delta=0,60)$. Em relação à sobrecarga subjetiva e criticismo as diferenças foram pequenas a médias, tendo melhores resultados na sobrecarga subjetiva. Em relação ao fator atitudes positivas, apesar de não termos obtido diferenças estatisticamente significativas, conseguimos aumentar estas atitudes nos familiares (aumento da média de 2,52 para 2,73), e obtemos diferenças estatisticamente significativas no momento pós intervenção em relação ao grupo de controlo.

No grupo de controlo não obtivemos diferenças significativas no pré e pós teste. Apesar de haver diferenças nas médias, estas são muito reduzidas, o que parece indicar que as diferenças obtidas no grupo experimental se podem dever à implementação do programa psicoeducacional, uma vez que ambos os grupos mantiveram as restantes intervenções que têm em comum.

Através da análise dos resultados, o programa parece ter conseguido diminuir a sobrecarga familiar e a emoção expressa, o que vai de encontro ao referido por diversos autores em relação à importância da psicoeducação familiar na redução da emoção expressa e sobrecarga familiar (Yacubian e Neto, 2001). Parece-nos ainda que a redução da sobrecarga familiar foi superior à redução da emoção expressa através da análise delta de Cliff. 
Estudos recentes semelhantes, como os de Sharif, Shaygan \& Mani (2012), Nasr \& Kausar (2009), Leal et al. (2008) e Cheng \& Chan (2005), encontraram resultados idênticos relativamente à redução da sobrecarga familiar com a aplicação de um programa psicoeducacional a familiares de utentes com esquizofrenia.

Relativamente à eficácia da psicoeducação familiar nas recaídas e reinternamentos, após um ano do final do programa a diferença entre o número de internamentos no grupo experimental foi muito inferior ao grupo de controlo, uma vez que apenas um utente do grupo dos familiares que foram submetidos ao programa psicoeducacional foi internado (10\%) e no grupo de familiares que não foram submetidos ao programa foram internados seis utentes (60\%), no mesmo período de tempo. Além disso, três destes utentes foram internados mais do que uma vez no espaço de um ano. Assim, parece-nos que a redução da emoção expressa e da sobrecarga nos familiares submetidos ao programa e a aquisição de competências para lidar com a doença levou à redução do número de internamentos, o que vai de encontro ao esperado.

\section{CONCLUSÕES}

Como conclusão, parece-nos que, a psicoeducação familiar é importante na reabilitação dos utentes com esquizofrenia, uma vez que como nos indicam Gonçalves-Pereira et al. (2007) e McFarlane et al. (2003), os conhecimentos recentes acerca dos mecanismos etiológicos da esquizofrenia mostram que, não só o tratamento, mas também o ambiente em que o doente vive e o tipo de estimulação que recebe, são determinantes na forma como a doença evolui e no nível de recuperação social que é atingido. Hervás (2008) acrescenta que se obtêm melhores resultados quando a abordagem da doença é feita de forma integrada, incluindo intervenções familiares. Além disso, conforme referem diversos autores como Brito (2006), Falloon et al. (1987), Gonçalves-Pereira et al. (2007), Lehman, et al. (2004) e McFarlane et al. (2003), a psicoeducação familiar têmse revelado muito eficaz na prevenção de recaídas e dos reinternamentos, contribuindo para melhorar a recuperação das pessoas com esquizofrenia e para preservar a saúde física e mental dos seus familiares, bem como a qualidade de vida. Através da análise da revisão da literatura e dos resultados desta investigação, parecenos crucial que as famílias façam parte integrante do processo de reabilitação do utente com esquizofrenia, devendo ser consideradas como uma peça fundamental deste puzzle incontornável que compõe esta perturbação mental grave.
Mais importante que o conhecimento da doença proporcionado pelo programa de psicoeducação, é a aliança terapêutica estabelecida entre o profissional e o familiar e o sentirem que não estão sozinhos nesta longa caminhada e que não são os únicos a viver este problema. Estudos futuros poderão analisar de forma mais aprofundada esta relação entre relação terapêutica, programas psicoeducativos e sobrecarga.

Estamos conscientes das limitações inerentes a este tipo de estudo, nomeadamente a dificuldade da homogeneização dos grupos experimentais e de controlo como se verificou neste estudo, apesar da selecção da amostra ter sido aleatória. Além disso e como nos referem Gonçalves-Pereira et al. (2006) uma das desvantagens das intervenções familiares a utentes com esquizofrenia em grupo é a fraca taxa de adesão. Esta foi, sem dúvida, a maior dificuldade sentida na elaboração deste estudo de investigação, uma vez que as perspetivas seriam obter uma amostra maior. A maioria dos motivos de recusa verbalizados pelos familiares foi o fator económico. Para colmatar esta entrave uma das hipóteses é a realização de sessões de psicoeducação mais próximas da comunidade, envolvendo as equipas de saúde mental comunitária. Para finalizar, sugerimos que em futuros estudos de investigação, se avaliem os motivos que levam os familiares a não aderirem a estes programas de intervenção para que depois possam ser analisadas estratégias de intervenção.

Gostaríamos ainda de acrescentar que, o facto da maior parte dos cuidadores informais dos utentes com esquizofrenia serem os seus pais e estes serem dependentes financeiramente, levanta uma questão relevante: "Qual o destino dos utentes com esquizofrenia quando não tiverem família?”. Esta é uma questão que deve preocupar o sistema nacional de saúde, ficando aqui mais uma sugestão para um estudo de investigação futuro.

\section{IMPLICAÇÕES PARA A PRÁTICA CLÍNICA}

Estes resultados podem ser cruciais para a prática clínica na medida em que reforçam a importância das intervenções familiares como parte integrante do processo de reabilitação de utentes com esquizofrenia. Assim, é fundamental que as instituições de saúde com unidades de psiquiatria implementem programas de reabilitação na área da esquizofrenia tendo em conta a intervenção familiar. Reabilitar o utente com esquizofrenia sem incluir intervenções que reduzam a emoção expressa e a sobrecarga da família pode tornar-se inútil quando o programa de reabilitação terminar e o utente voltar para o seio familiar. Todos os progressos reabilitativos devem ter em conta o regresso ao lar. 
Não só o utente, mas também a família deve aprender a lidar com a doença mental grave para poderem crescer em conjunto e lutar contra as intempéries que a esquizofrenia acarreta.

\section{REFERÊNCIAS BIBLIOGRÁFICAS}

American Psychiatric Association (2014). DSM-V Manual de Diagnóstico e Estatística das Perturbações Mentais (5a ed.). Lisboa: Climepsi Editores.

Awad, A. G., \& Voruganti, L. N. (2008). The burden of schizophrenia on caregivers. Pharmacoeconomics, 26(2), 149-162.

Borba, L. O., Schwartz, E., \& Kantorski, L. P. (2008). A sobrecarga da família que convive com a realidade do transtorno mental. Acta Paulista de Enfermagem, 21(4), 588-594.

Brito, M. L. S. (2006). Intervenções psicoeducativas para famílias de pessoas com esquizofrenia: Revisão sistemática da literatura. Revista Portuguesa de Enfermagem, 5, 15-24.

Cheng, L. Y., \& Chan, S. (2005). Psychoeducation program for Chinese family carers of members with schizophrenia. Western Journal of Nursing Research, 27(5), 583-599.

Falloon, I. R. H., McGill, C. W., Boyd, J. L., \& Pederson, J. (1987). Family management in the prevention of morbidity of schizophrenia: Social outcome of a twoyear longitudinal study. Psychological Medicine, 17, 59-66.

Gonçalves-Pereira, M., Xavier, M., e Fadden, G. (2007). O Modelo de Falloon para intervenção familiar na esquizofrenia: Fundamentação e aspectos técnicos. Análise psicológica, 25(2), 241-255.

Gonçalves-Pereira, M., Xavier, M., Neves, A., Barahona-Correa, B., e Fadden, G. (2006). Intervenções familiares na esquizofrenia: dos aspectos teóricos à situação em Portugal. Acta Médica Portuguesa, 19(1), 1-8.

Hervás, M. D. C. (2008). Rehabilitación y psicoeducación en la esquizofrenia. In A. C. Moreno, Las esquizofrenias - sus hechos y valores clínicos y terapêuticos. Barcelona: Elsevier Masson.
Leal, M., Sales, R., Ibáñez, E., Giner, J., \& Leal, C. (2008). Valoración de la sobrecarga en cuidadores informales de pacientes con esquizofrenia antes y después de un programa Psicoeducativo. Actas Españolas de Psiquiatria, 6(2), 63-69.

Lehman, A. F., Lieberman, J. A., Dixon, L. B., McGlashan, T. H., Miller, A. L., Perkins, D. O., \& Kreyenbuhl, J. (2004). Practice guideline for the treatment of patients with schizophrenia. The American Journal of Psychiatry, 161(2), 1-56.

Lucksted, A, McFarlane, W., Downing, D., \& Dixon, L. (2012). Recent developments in family psychoeducation as an evidence-based practice. Journal of Marital and Family Therapy, 38(1), 101-121. doi: 10.1111/j.17520606.2011.00256.x

McFarlane, W. R., Dixon, L., Lukens, E., \& Lucksted, A. (2003). Family psychoeducation and schizophrenia: A review of the literature. Journal of Marital and Family Therapy, 29(2), 223-245.

Nasr, T., \& Kausar, R. (2009). Psychoeducation and the family burden in schizophrenia: A randomized controlled trial. Annals of General Psychiatry, 8, 17. doi: 10.1186/1744-859X-8-17. Acedido em http://www.annals-general-psychiatry.com/content/8/1/17

Pfammatter, M., Andres, K., e Brenner, H. (2012) Manual de psicoeducação e gestão da doença na esquizofrenia. Edição Encontrar-se.

Reis, F. (2004). Intervenção psicoeducativa nas famílias de wsquizofrénicos. Revista do Serviço de Psiquiatria do Hospital Fernando Fonseca, 1(1), 19-41.

Sharif, F., Shaygan, M., \& Mani, A. (2012). Effect of a psycho-educational intervention for family members on caregiver burdens and psychiatric symptoms in patients with schizophrenia in Shiraz, Iran. BMC Psychiatry, 12, 48. doi:10.1186/1471-244X-12-48. Acedido em http://www.biomedcentral.com/1471-244X/12/48

Xavier, M., Baptista, H., Mendes, J., Magalhães, P., e Caldas-de-Almeida, J. (2013). Implementing the World Mental Health Survey Initiative in Portugal - rationale, design and fieldwork procedures. International Journal of Mental Health Systems, 7(19). Acedido em http:// www.ijmhs.com/content/7/1/19 
Xavier, M., Pereira, M. G., Corrêa, B. B., e Caldas-deAlmeida, J. (2002). Questionário de problemas familiares: Desenvolvimento da versão portuguesa de um instrumento de avaliação de sobrecarga familiar. Psicologia, Saúde \& Doenças, 3(2), 165-177.
Yacubian, J., e Neto, F. L. (2001). Psicoeducação familiar. Família, Saúde e Desenvolvimento, 3(2), 98-108.

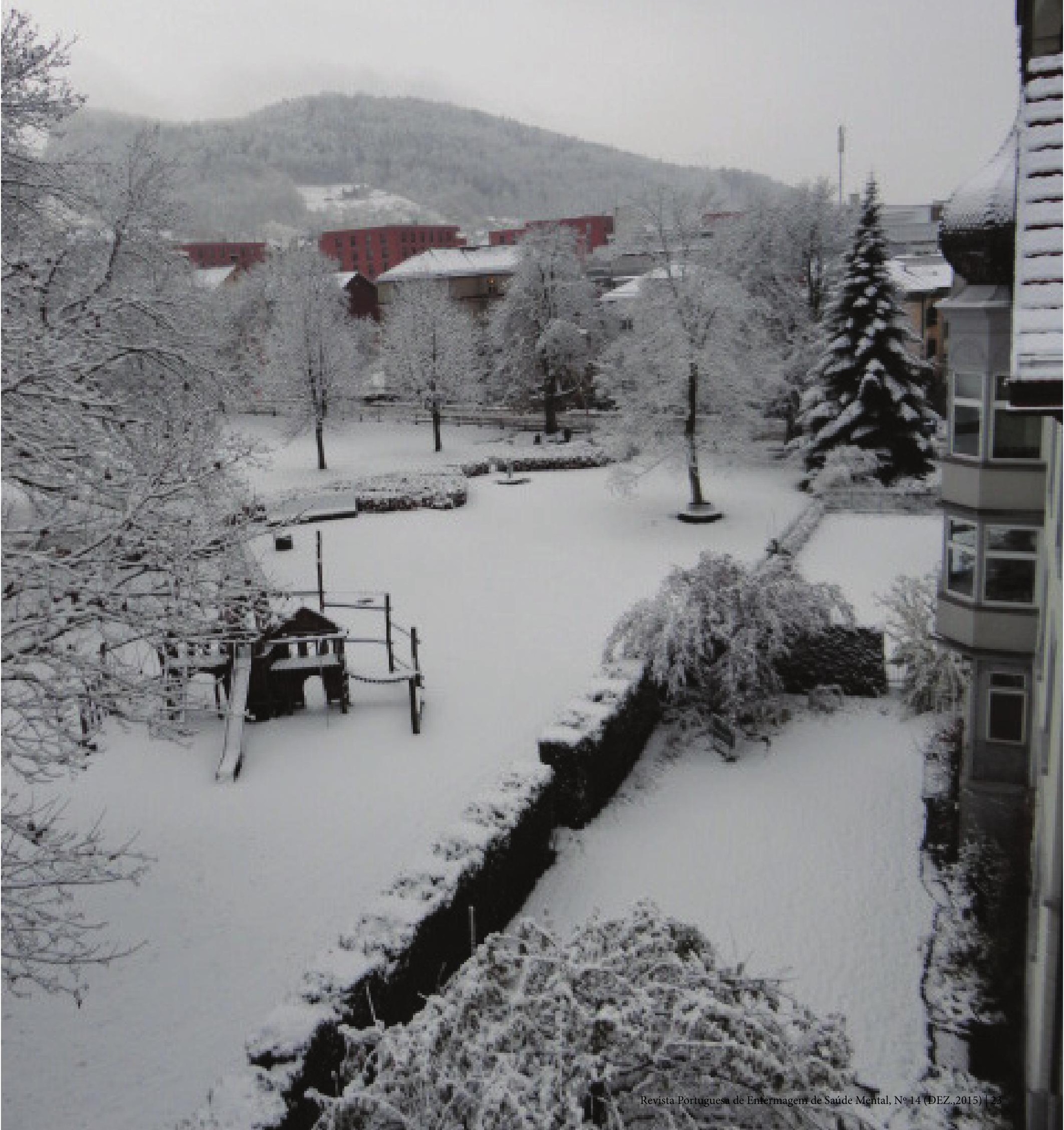

